\title{
Defining a Molecular Atlas of the Hippocampus Using DNA Microarrays and High-Throughput In Situ Hybridization
}

\author{
Ed S. Lein, ${ }^{1}$ Xinyu Zhao, ${ }^{2}$ and Fred H. Gage ${ }^{1}$ \\ ${ }^{1}$ Laboratory of Genetics, The Salk Institute for Biological Studies, La Jolla, California 92037, and 2Department of Neurosciences, University of New Mexico, \\ Albuquerque, New Mexico 87131
}

\begin{abstract}
The hippocampus consists of a series of cytoarchitecturally discrete subregions that can be distinguished from one another on the basis of morphology, connectivity, and electrophysiological properties. To understand the molecular underpinnings for these differences, DNA microarrays were used to find genes predicted to be enriched in subregions CA1, CA3, and the dentate gyrus, and $>100$ of these genes were subsequently analyzed using in situ hybridization to obtain cellular-level localization of their transcripts. The most striking commonality among the resulting patterns of gene expression is the extent to which cytoarchitectural boundaries within the hippocampus are respected, although the complexity of these patterns could not have been predicted on the basis of the microarray data alone. Among those genes with expression that can be characterized as "restricted" to neurons in one or more subregions of the hippocampus are a number of signal transduction molecules, transcription factors, calcium-binding proteins, and carbohydrate-modifying enzymes. These results suggest that important determinants of the unique identities of adult hippocampal neurons are differential signal transduction, regulation of gene expression, calcium homeostasis, and the maintenance of a unique extracellular milieu. Furthermore, the extremely high correlation between microarray data and in situ expression demonstrates the great utility of using DNA microarrays to genetically profile discrete brain regions.
\end{abstract}

Key words: hippocampus; in situ hybridization; DNA microarray; expression profiling; dentate gyrus; CA2; CA1; CA3

\section{Introduction}

The hippocampus is one of the most thoroughly characterized regions of the mammalian brain, in large part because of its highly distinctive morphology (Ramon y Cajal, 1911; Lorente de Nó, 1934), its critical role in learning and memory (Zola-Morgan et al., 1986; Scoville and Milner, 2000), and because it is one of the few regions in the adult brain where ongoing neurogenesis is observed (Altman and Das, 1965; Kempermann et al., 1997). Historically, the hippocampus has been divided into two main regions: the dentate gyrus (DG), consisting primarily of granule cells, and Ammon's horn, consisting of excitatory pyramidal neurons that can be divided into CA1, CA2, and CA3 subregions (Lorente de Nó, 1934). Each of these neuronal populations can be distinguished on the basis of morphology, connectivity, electrophysiological properties, and susceptibility to insults (Lorente de Nó, 1934; Corsellis and Bruton, 1983; Amaral and Insausti, 1990).

Excitatory neurons in each subregion of the hippocampus are also molecularly distinct. Many studies of gene expression in em-

\footnotetext{
Received 0ct. 17, 2003; revised March 5, 2004; accepted March 8, 2004.

This research was supported by National Institute on Aging Grant AG06088, The Christopher Reeves Paralysis Foundation, a grant from the David and Lucile Packard Foundation, and National Institutes of Health Bioengineering Research Partnership Grant EY14103. We thank Dr. C. Aston and Wyeth Neuroscience for assistance with the initial microarray data. We are also grateful to Tara Martinez for expert technical assistance and J. Nathanson, Dr. E. Callaway, and Dr. L. Stefanacci for their critical reading of this manuscript.

Correspondence should be addressed to Fred H. Gage, The Salk Institute for Biological Studies, Laboratory of Genetics, P.0. Box 85800, San Diego, CA 92186-5800. E-mail: gage@salk.edu.

DOI:10.1523/JNEUROSCI.4710-03.2004

Copyright $\odot 2004$ Society for Neuroscience $\quad$ 0270-6474/04/243879-11\$15.00/0
}

bryonic (Tole et al., 1997) and adult (Zhao et al., 2001) animals have demonstrated striking region-specific gene expression. This finding suggests that such discrete regulation of gene expression underlies functional differences between these neuronal populations. With the advent of genome-scale molecular techniques, it has become possible to profile global expression patterns in discrete tissues. These techniques [microarrays, serial analysis of gene expression (SAGE)] have been applied to the hippocampus as a complete structure in the developing and adult rodent (Datson et al., 2001; Mody et al., 2001). These techniques hold great promise for understanding hippocampal structure and function, but the resulting data are of limited utility if the exact cells in which genes are expressed are not known. A systematic approach to examining hippocampal gene expression at cellular-level resolution will provide data that are of immediate utility in describing functional differences between neuronal subtypes and potentially in allowing specific genetic manipulation of those cells.

To further understand differences between hippocampal subtypes, we used microarrays to perform expression profiling on microdissected hippocampal regions CA1, CA3, and the dentate gyrus. A previous report on the early stages of this project demonstrated that the expression of a small set of genes is restricted to CA1, CA2, CA3, or the dentate gyrus (Zhao et al., 2001). In the current study, more relaxed analytical criteria were used on a larger data set to extend the list of candidate genes predicted to be enriched in each hippocampal subregion, and a methodology for high throughput in situ hybridization was used to examine the expression patterns of $>100$ genes predicted to have the highest 
(or lowest) expression in one of these regions. The goals of the present study are twofold: (1) to examine the fidelity of DNA microarrays by systematically confirming expression patterns of a large set of genes predicted to be enriched in each hippocampal subregion, and (2) more importantly, to develop a molecular "atlas" of the hippocampus on the basis of subregion-specific patterns of gene expression, with the ultimate goal of understanding functional differences between cell types in these regions.

\section{Materials and Methods}

Animals and tissue. All animal procedures were performed according to protocols approved by The Salk Institute for Biological Studies Animal Care and Use Committee. Ten- to 11-week-old C57BL/6 male mice were obtained from Harlan (San Diego). Mice were killed by intraperitoneal injection of a mixture of ketamine $(75 \mathrm{mg} / \mathrm{kg})$, xylazine $(4 \mathrm{mg} / \mathrm{kg})$, and acepromazine $(5.6 \mathrm{mg} / \mathrm{kg})$. For DNA microarray analysis, brains were rapidly dissected, and the hippocampi were removed and subdissected to yield CA1, CA3, and dentate gyrus tissues (supplemental Fig. 1; available at www.jneurosci.org), which were immediately frozen on dry ice. Eleven groups of animals were processed independently, with each group consisting of tissue pooled from five to seven animals. For in situ hybridization, brains were rapidly dissected, rinsed in ice-cold $0.1 \mathrm{~m}$ phosphate buffer, frozen in optimal cutting temperature mounting medium (TissueTek; Sakura Finetek, Torrance, CA) in a dry ice-isopentane slurry, and stored at $-80^{\circ} \mathrm{C}$ until use.

RNA preparation and array hybridization. Total RNA was isolated from hippocampal tissue using Trizol (Invitrogen, Gaithersburg, MD). Sample labeling, hybridization, and scanning were performed as described previously (Wodicka et al., 1997). Affymetrix Murine11K subA and subB array sets were used for all hybridizations (Affymetrix, Santa Clara, CA). Each set contained 13,069 probe sets corresponding to $\sim 11,000$ individual genes and expressed sequence tags (ESTs) from Unigene and TIGR (Lockhart and Winzeler, 2000). A total of 33 samples (CA1, CA3, and DG samples from 11 groups of animals) was hybridized to 33 chip sets (subA and subB) in this study. Some of these animals had been allowed to run on running wheels, but none of the genes described in this study showed consistent changes associated with running. Data from these animals can therefore be considered equivalent to those obtained from animals housed in standard conditions, and the inclusion of these data greatly enhanced our ability to search for consistent intrahippocampal expression patterns across many data sets. Correlation coefficients between individual microarrays were often quite low, ranging from 0.81 to 0.99 . However, within a given experimental data set consisting of CA1, CA3, and DG samples generated from the same animals, the correlation coefficients between samples were much higher, generally between 0.95 and 0.98 .

Data analysis. Initial data analysis was performed using Affymetrix Genechip version 3.3 software, and expression levels [average difference $(A D)$ values] were normalized to a target intensity of 2500 for each array. $\mathrm{AD}$ values [without reference to present calls or fold changes (FCs)] for all 33 data sets ( 11 each for CA1, CA3, and DG) were then filtered using Genespring software (Silicon Genetics, Redwood City, CA). The "drawn gene" function in Genespring was used to search for trends in gene expression across all data sets by searching for genes displaying a high correlation coefficient to a model expression pattern highest (or lowest) in one region relative to the others (see Fig. 1). The resulting rankordered gene lists for a given pattern (e.g., highest in DG) were then visually examined to determine the strengths of the matches, and these candidates were also compared with gene lists generated from our previous study on a subset of these data using high-stringency data analysis (Zhao et al., 2001). Gene lists generated using the present data analysis method included almost all of the genes previously identified using Affymetrix present calls and FC cutoffs, although this method was far more inclusive, and the rank ordering was very different. Expression data found in the literature confirmed predicted expression patterns for many genes, and a total of 104 candidate genes was selected for analysis by in situ hybridization. The tabulated results for genes described in the liter- ature, in addition to those directly assessed by in situ hybridization, are detailed in supplemental Table 1 (available at www.jneurosci.org). Nomenclature from Mouse Genome Informatics (MGI) is used throughout to standardize nomenclature. Functional annotation used in this manuscript is based on gene ontology (GO) listings (Ashburner et al., 2000) derived from MGI (http://www.informatics.jax.org/) (Blake et al., 2003), Affymetrix (Liu et al., 2003), and Swiss-Prot/TrEMBL (http://us.expasy. org/sprot/) (Appel et al., 1994) databases.

In situ hybridization. Clones for the genes analyzed were either ordered from the I.M.A.G.E. Consortium (Incyte Genomics, Palo Alto, CA) or amplified by PCR from mouse brain cDNA and cloned into the pCRIITOPO cloning vector (Invitrogen) (see supplemental material for EST clone identification and PCR primers used to generate clones; available at www.jneurosci.org). Sequence-confirmed plasmids were linearized and used for the synthesis of sense and antisense $\alpha-{ }^{35}$ S-labeled riboprobes using T3, T7 (Stratagene, La Jolla, CA), or Sp6 (Promega, Madison, WI) RNA polymerases. In situ hybridization was performed essentially as described previously (Lein et al., 2000), although methodology was developed to allow the processing of large numbers $(\sim 150)$ of slides simultaneously. Hemispheres from three different animals were prepared as described above in a single block, allowing all three to be cut simultaneously near the same plane of section to assess reproducibility across animals. Twelve micrometer cryostat sections through the entire hippocampal formation of each set of brains were cut and mounted on Superfrost slides (Sigma, St. Louis) in such a way that slide 1 contained sections 1, 101, and 201, slide 2 contained sections 2, 102, and 202, and so on. Each probe was hybridized to three evenly spaced slides (e.g., slides 1, $33,66)$, such that in situ hybridization data were obtained for each probe at nine planes of section through the hippocampus in triplicate. Processing in this way allowed a very detailed analysis of gene expression throughout the entire hippocampal formation. Sense controls were performed for a subset of genes and in all cases yielded nonspecific background labeling (data not shown).

After in situ hybridization, slides were first exposed to X-ray film (XOMAT AR; Eastman Kodak, Rochester, NY), subsequently defatted and dipped in autoradiographic emulsion (NTB2; Eastman Kodak), and allowed to expose for 1 and one-half to 6 weeks, depending on the strength of labeling observed on $\mathrm{x}$-ray film. Slides were then developed and stained with the fluorescent dye bisbenzimide (Sigma) to allow localization of silver grains to individual cells. Slides of in situ hybridization were digitized using a cooled CCD camera (SPOT2; Diagnostic Instruments, Sterling Heights, MI) mounted on a Nikon Eclipse TE300 microscope (Nikon, Melville, NY) using an external dark-field adapter (Darklite Vertical; Micro Video Instruments, Avon, MA). Contrast levels were adjusted in Adobe Photoshop (Adobe Systems, San Jose, CA).

\section{Results}

\section{Tissue isolation and candidate selection}

To obtain tissue for microarray analysis, hippocampal subregions were isolated from freshly dissected 10-week-old C57BL/6 mice (supplemental Fig. 1; available at www.jneurosci.org). First, the entire hippocampus was dissected away from the overlying neocortex. The natural division of the hippocampal fissure marks the boundary between Ammon's horn and the dentate gyrus, making it possible to isolate the top portion of Ammon's horn (approximately CA1). The remainder of Ammon's horn (approximately CA3) can then be dissected away from the dentate gyrus, the boundaries of which are clearly visible. It should be noted that, given the gross nature of this dissection, the hilus of the dentate gyrus and most likely the proximal portion of CA3 (CA3c) are included in the dentate gyrus tissue sample. Furthermore, to fully understand the results of this screen, it is also important to note the structures adjacent to different subregions of the hippocampus. First, the main target structure of CA1 projections, the subiculum, lies immediately adjacent to CA1, and the boundary between CA1 and the subiculum is not easily discerned in fresh tissue. Therefore, it is likely that some or all of the subiculum is 


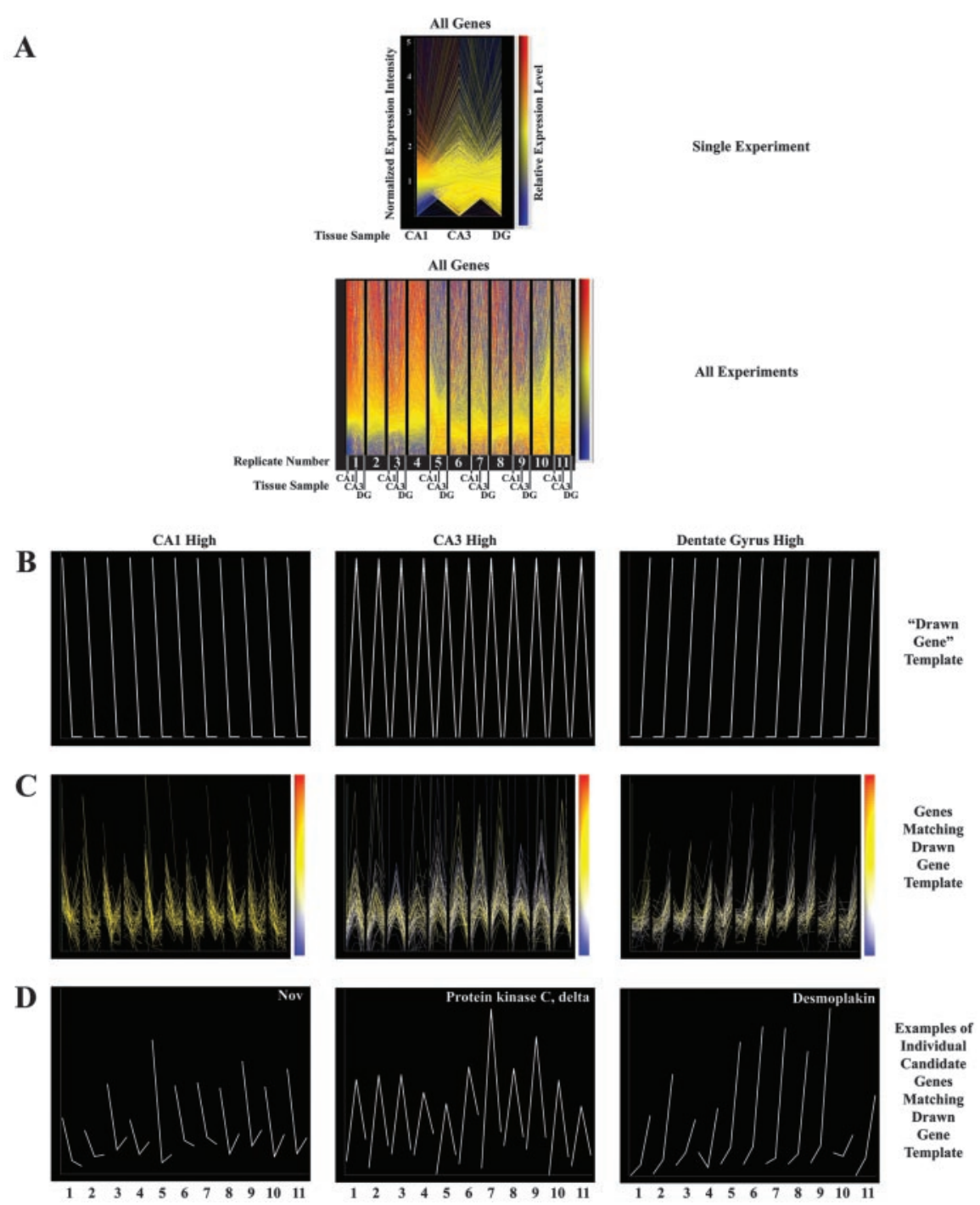

Figure 1. Schematic of microarray data analysis. Data from regions CA1, CA3, and the dentate gyrus of each individual set of animals were grouped together to assess relative differences in gene expression across these regions within a single experiment ( $A$, top panel). Expression levels are plotted as a continuous line for a particular gene, normalized to the median value of all genes on that microarray and to the median value for that gene across all microarrays, and color coded for the expression level of that gene relative to all other genes. Data from all 11 experiments are plotted in the bottom panel in $A$. B, Data from all 11 independent replicates were probed simultaneously for genes matching a particular pattern of expression (highest in CA1, highest in CA3, or highest in DG). The subset of genes matching this pattern are plotted in C, and one individual gene matching each pattern across all 11 replicates is plotted in $D$.

included in the CA1 tissue sample. Similarly, the fimbria and choroid plexus are adjacent to CA3 throughout the entire septotemporal length of the hippocampus and, given the necessity for a rapid dissection to ensure the isolation of intact RNA, they could not be fully removed from the CA3 sample.

Tissue from five to seven mice was pooled for each sample to minimize variability in the dissection and to obtain sufficient material to use for microarray analysis. Results from the first two samples generated have been described previously (Zhao et al., 2001). Nine additional independent replicates were generated and pooled with the previous data for subsequent data analysis. With only two replicates, the options for data analysis were limited to pairwise comparisons using threshold cutoffs for expression levels and FCs between samples (Zhao et al., 2001). With larger numbers of replicates, it was possible to use other methods to search for more subtle trends in the data. An analysis method in Genespring software (Silicon Genetics) was used to search for genes with overall expression patterns across all replicates that matched a fabricated pattern by simply calculating the correlation coefficient between the $\mathrm{AD}$ values and the fabricated pattern. The resulting list of candidate genes was much larger than that obtained with FC cutoffs or present calls ( $>50$ candidates per set vs 6-15 using cutoffs) and almost completely contained the smaller set of genes previously identified using two data sets and threshold cutoffs. Two of the candidate genes from the smaller data set (Zhao et al., 2001) did not demonstrate the same pattern of expression across all 11 data sets and can be considered false positives (L42340, $\mathrm{Na}^{+}$channel 27; C76099, EST). Furthermore, many genes with the same trend across the entire data set but with widely varying FCs, low expression values, or several outliers were identified as strong candidates.

The data analysis scheme is shown in Figure 1. Data from all 33 microarrays were analyzed simultaneously by searching for genes matching a fabricated template pattern, in this case a gene highly enriched in one of the subregions (CA1, CA3, or DG) relative to the others. A threshold correlation coefficient determined the necessary strength of the match and determined the number of candidate genes generated, rank ordered by the correlation coefficient describing the degree of similarity to the template pattern. The ranking for each gene examined is listed in supplemental Table 1 (available at www.jneurosci.org). A 10-fold enrichment in one hippocampal subregion relative to the others was used to generate lists of candidate genes. A small additional set of genes that were not picked up with the method described above was generated using a drawn gene template with 40 -fold enrichment in one sample. These genes tended to be expressed at low levels and had negative values for nonexpressing regions, thus yielding very large apparent FCs. Threshold correlation coefficients between 0.80 and 0.85 generated between 50 and 70 candidate genes that were predicted to be enriched in one hippocampal subregion relative to the others. It should be noted that the great majority of candidate genes had actual FCs of less than threefold between regions. Thus, the low correlation coefficients obtained were a consequence of setting the target enrichment artificially high. Interestingly, the opposite approach, using this method to search for genes lowest in one area by setting two of the three tissue types to 40 -fold higher than the third, was much less successful at pulling out promising candidate genes, and most of these candidates overlapped with genes predicted to be highest in one region.

To pick candidate genes to verify using in situ hybridization, the relative expression patterns of each gene were examined visually for the consistency of the predicted pattern of expression, the 
expression level predicted by the microarray (AD value), and the FCs between hippocampal subregions. In addition, we searched for archival expression data preexisting in the literature for genes in these lists. The vast majority of candidate genes that could be identified in the literature demonstrated regional variation within the hippocampus consistent with our data (see supplemental Table 1; available at www.jneurosci.org), justifying the selection of the majority of remaining candidates for further characterization by in situ hybridization. The microarray data for several exemplar genes are plotted in the bottom panel of Figure 1 , demonstrating that some of the genes from each category showed remarkable consistency across all 11 data sets, although there was considerable variability in the FCs between experiments.

A standard in situ hybridization protocol (Lein et al., 2000) was scaled up to allow simultaneous processing of large numbers of slides and probes. The predicted expression levels ( $\mathrm{AD}$ values) of the genes examined ranged over two orders of magnitude, from $\sim 300$ to 60,000 . Despite this range, the same methodology worked well on all probes used, without any modification of washing stringency or hybridization temperature. After in situ hybridization, the slides were placed on $\mathrm{x}$-ray film to ascertain the labeling intensity and to help decide how long to expose the slides on photographic emulsion. In a very general sense, this labeling intensity correlated with the predicted $\mathrm{AD}$ values. However, in individual cases, the $\mathrm{AD}$ value was not a very accurate predictor of expression level, as assessed by in situ hybridization. In addition, in some cases, there were multiple probe sets for the same gene on the microarrays, and the two probe sets yielded $\mathrm{AD}$ values differing from one another by an order of magnitude. Although it is possible that this discrepancy is attributable to splice variation between portions of the gene probed with the different probe sets, it seems likely that some probe sets work better than others, and $\mathrm{AD}$ values should therefore only be taken as a general indication of actual expression levels.

The remainder of this study describes the variety of expression patterns observed among a large set of genes predicted to have the highest (or lowest) levels of expression in CA1, CA3, or the dentate gyrus of the hippocampus. Remarkably, 100 of 104 genes examined demonstrated patterns of expression that were consistent with the predicted patterns. Within the hippocampus, the overwhelming feature was the degree to which boundaries of gene expression respected the cytoarchitectural boundaries defining CA1, CA2, CA3, and DG. However, the classification scheme of "CA1 highest," "CA3 highest," and "DG highest" proved insufficient to describe the complexity of the data. Therefore, the description of the expression patterns observed has been grouped together by more salient identifiers.

\section{Definition of boundaries between hippocampal subregions}

A subset of genes predicted to be highest in CA1, CA3, or the dentate gyrus proved to be restricted to these regions. A set of exemplar genes in Figures 2 and 3 demonstrates robust subregion-specific gene expression in the primary excitatory neuronal populations that serve to identify the different cytoarchitectural boundaries used for the remainder of the manuscript. In situ hybridization signal for nephroblastoma-overexpressed gene (Nov) is restricted to the CA1 pyramidal layer (Fig. 3A,E) (Zhao et al., 2001). CA3 is uniquely delimited by expression of bcl-2-related ovarian killer protein (Fig. $2 U, Z$ ). Finally, expression of Purkinje cell protein 4 is restricted to the dentate gyrus and CA2 (Fig. 3Q,U) as described previously (Zhao et al., 2001). Together, these three genes tile the major excitatory cell popula- tions of the hippocampus and serve to define the boundaries of each subregion. These boundaries of gene expression perfectly match cytoarchitectural boundaries observed by cresyl violet staining (data not shown), with the exception of the CA2/CA3 boundary, which is not possible to see using standard histological stains. All of the slides of in situ hybridization were also stained with the fluorescent dye bisbenzimide, which stains nuclei. At least in tissue processed for in situ hybridization, this stain very clearly delimits the CA1/CA2 boundary throughout the septotemporal axis of the hippocampus and is used throughout this study to define this boundary in the same tissue sections processed for in situ hybridization.

The majority of genes examined demonstrate more complex patterns of expression than those described above. First, some genes are expressed in only one (or several) subregion of the hippocampus and can be classified as "restricted" to that region (or regions). Many genes are high in one (or more) region and relatively uniformly expressed in the others and can therefore be classified as "enriched" in that region (or regions). Although this classification is somewhat arbitrary in that there may be issues of sensitivity or background related to in situ hybridization leading to a classification of enriched or restricted, it is a useful classification to denote highly specific gene expression within particular cell types. Genes are classified into categories on the basis of expression within granule cells of the dentate gyrus and pyramidal cells in subregions CA1, CA2, or CA3 (and in one case, the hilus of the dentate gyrus). Expression within less prominent populations of cells such as interneurons, astrocytes, and oligodendrocytes adds another important level of complexity to the observed expression patterns but precludes clustering of genes into a small number of categories. This classification scheme is used in supplemental Table 1 (available at www.jneurosci.org), and the variety of combinations of restriction or enrichment is used to cluster patterns in Figures 2 and 3. These clusters are most easily ordered as reciprocal sets (e.g., highest in DG, lowest in DG) and are presented as such. A small set of genes selected to demonstrate the range of results is presented in Figures 2 and 3, and a larger set of genes displaying each expression pattern is available as supplementary material (available at www.jneurosci.org). Because the microarray data could only predict expression in the hippocampal regions directly arrayed, low-magnification images of the entire hemisphere are also included to allow an examination of the overall pattern of expression of that gene throughout the brain as well.

\section{Genes enriched in the dentate gyrus or lowest in the dentate gyrus}

Many genes predicted to be enriched in the dentate gyrus proved to be restricted to or heavily enriched in granule cells of the dentate gyrus (Fig. 2) (supplementary Fig. 2; available at www.jneurosci. org). At the extreme, desmoplakin, a component of desmosomal tight junctions in peripheral tissues (Kowalczyk et al., 1996), is completely restricted to granule cells of the dentate gyrus, both within the hippocampus (Fig. $2 A, F$ ) and throughout the entire brain (supplemental Fig. 12; available at www.jneurosci.org). This pattern of expression is completely consistent with the microarray data, which predicted more than a twofold enrichment in the dentate gyrus in all 11 data sets (Fig. 2P). It should be noted at this point that, in general, the degree of enrichment observed by in situ hybridization is greater than that predicted from microarray data, as reported by others (Zirlinger et al., 2001). Mini chromosome maintenance deficient 6, a gene involved in initiation of DNA replication in C. elegans, is also uniquely expressed in dentate gyrus granule cells relative to pyrami- 


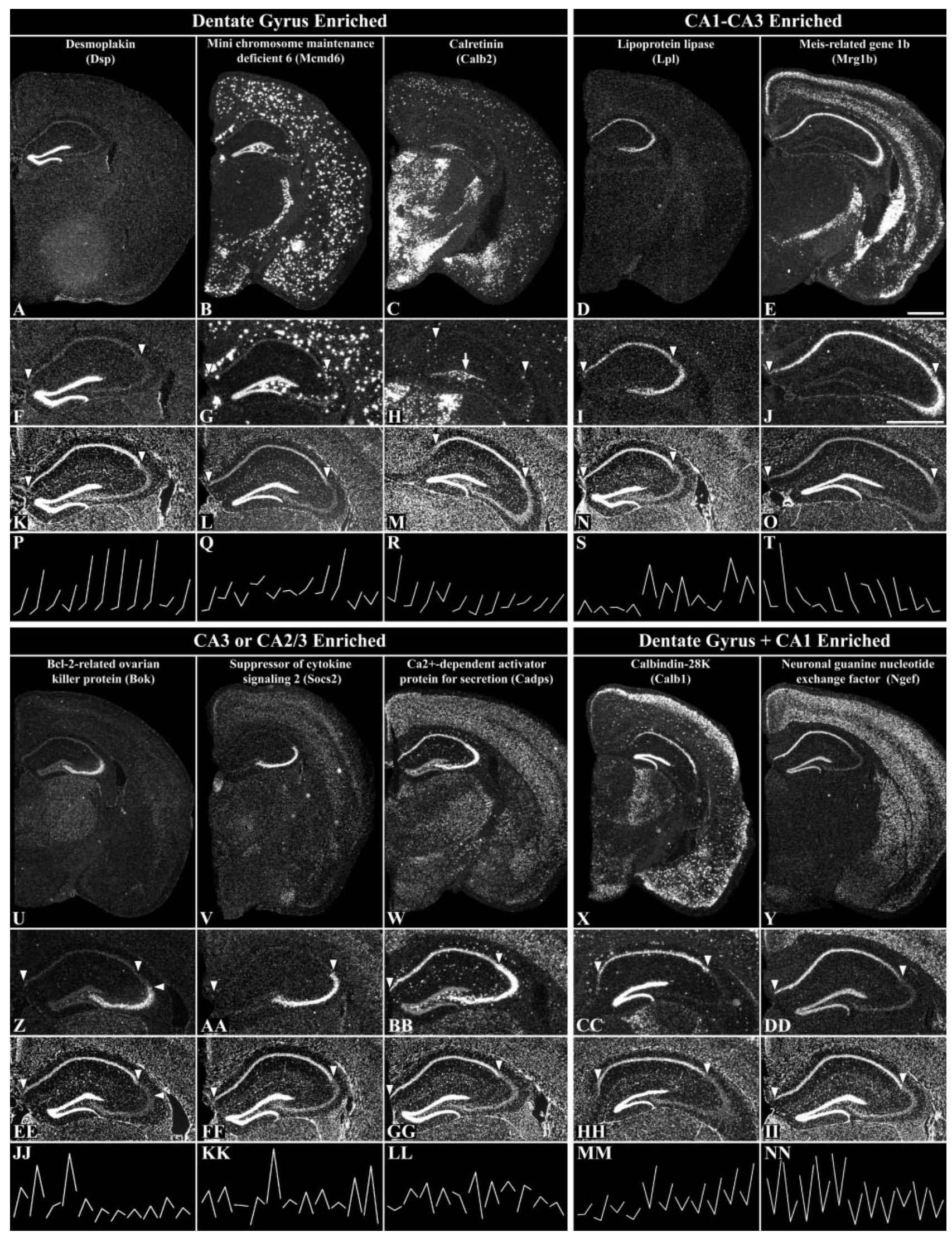

Figure 2. Subregion-specific patterns of gene expression within the hippocampus I. $A-E, U-Y$, Genes enriched in the dentate gyrus ( $A-C), C A 1-C A 3(D, E), C A 3$ or $C A 2$ plus $C A 3$ (CA2/3; $U-W)$, and the dentate gyrus plus $C A 1(X, Y) . A-J, U-D D$, In situ hybridization for $D s p(A, F), \operatorname{Mcmd6}(B, G)$, calretinin $(C, H), \operatorname{Lpl}(D, I), \operatorname{Mrg} 1 \mathrm{~b}(E, J), \operatorname{Bok}(U, Z) \operatorname{Socs} 2(V, A A)$, Cadps $(W, B B)$, calbindin $(X, C C)$, and $\operatorname{Ngef}(Y, D D)$ on coronal sections through the hippocampus at low magnification $(A-E, U-Y)$ and high magnification $(F-J, Z-D D)$. Bisbenzimide staining of the same sections is shown in $K-0$ and $E E-I I$, and gene chip data for each gene are shown in $P-T$ and $J J-N N$. Arrowheads in $F-O$ and $Z-I /$ delimit the boundaries of $C A 1$ and also the boundary between $C A 2$ and $C A 3$ in $Z$ and $E E$. The arrow in $H$ denotes the hilus of the dentate gyrus. Scale bars, $1 \mathrm{~mm}$. 
dal cells in Ammon's horn (Fig. 2B,G). However, it is also very highly expressed in interneuronal populations in the hilus, stratum oriens of Ammon's horn, and throughout most of the brain. Finally, calretinin, best known as a marker for subpopulations of inhibitory cells (Miettinen et al., 1992; Gonchar and Burkhalter, 1997), is also heavily enriched in the dentate gyrus (Fig. $2 \mathrm{C}, \mathrm{H}$ ), as predicted by the microarray data. However, expression is limited to cells within the hilus and subgranular zone (SGZ) of the dentate gyrus, and no label is seen within the granule cell layer.

Another prominent population of genes is expressed in all pyramidal cells and excluded from granule neurons of the dentate gyrus (Fig. 2) (supplementary Fig. 3; available at www.jneurosci.org). Genes falling into this category were either predicted to be highest in CA3 (Fig. 2S), highest in CA1 (Fig. 2T), or lowest in the dentate gyrus (supplemental Fig. 3; available at www.jneurosci.org), depending on the relative expression levels within CA1 and CA3. For example, lipoprotein lipase is expressed throughout the pyramidal cell layer with no expression in dentate gyrus granule cells (Fig. 2D,I) and is nearly restricted to the hippocampus relative to the rest of the brain. Expression of the transcription factor mrglb is restricted to pyramidal cells in CA1-CA3 as well, although labeling does not extend into the portion of CA3 most proximal to the dentate gyrus (Fig. 2E,J).

Genes enriched in CA3 or CA2 plus CA3 or lowest in CA2 plus CA3

A large number of genes predicted to be enriched in CA3 was confirmed to be enriched in this structure (Fig. 2) (supplementary Fig. 4; available at www.jneurosci.org). However, two categories of genes emerged from a closer analysis. Several genes are expressed solely within CA3, including bcl-2related ovarian killer protein (Fig. $2 U, Z$ ). In the majority of cases, however, expression is present in both CA2 and CA3 (CA2/3). For example, suppressor of cytokine signaling 2 (Fig. $2 V, A A$ ) is restricted to CA2/3 within the hippocampus, whereas $\mathrm{Ca}^{2+}$-dependent activator protein for secretion (Fig. $2 W, B B$ ) is enriched in $\mathrm{CA} 2 / \mathrm{CA} 3$.

A relatively small set of genes was expressed in the reciprocal pattern, with highest levels in the dentate gyrus and CA1 and with lower or no expression in CA2/3 (Fig. 2) (supplementary Fig. 5; available at www.jneurosci.org). Genes falling into this category were either predicted to be highest in the dentate gyrus (Fig. 2MM), lowest in CA3 (Fig. 2NN), or highest in CA1 (supplemental Fig. 5; available at www.jneurosci.org), again depending on the relative expression levels in the dentate gyrus and CA1.
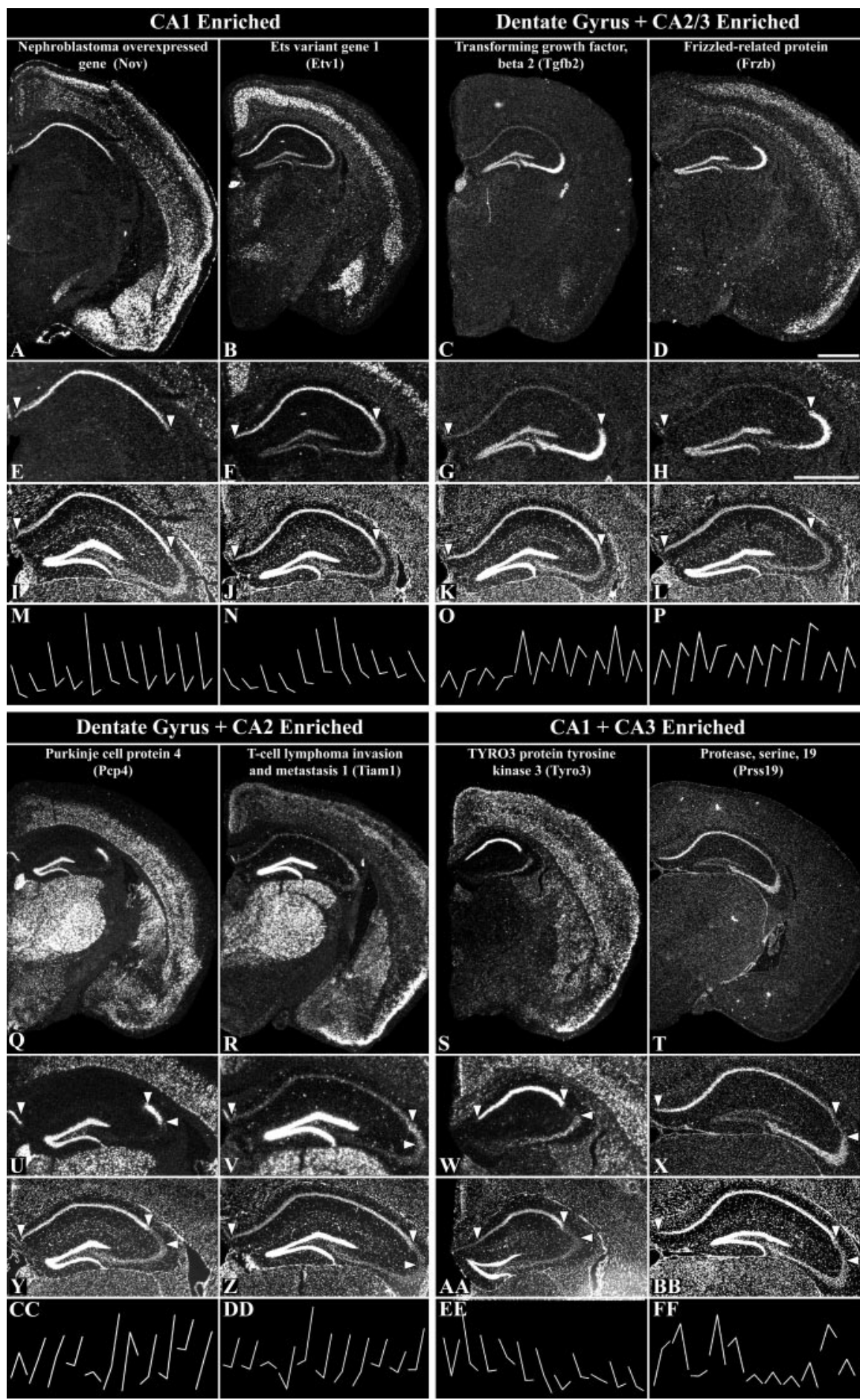

Figure 3. Subregion-specific patterns of gene expression within the hippocampus II. $A-D, Q-T$, Genes enriched in $C A 1(A, B)$, the dentate gyrus plus $C A 2 / 3(C, D)$, the dentate gyrus plus $C A 2(Q, R)$, and $C A 1$ plus $C A 3(S, T) . A-H, Q-X$, In situ hybridization for Nov $(A, E), \operatorname{Etv} 1(B, F), \operatorname{Tgfb2}(C, G), \operatorname{Frzb}(D, H), \operatorname{Pcp} 4(Q, U), \operatorname{Tiam} 1(R, V), \operatorname{Tyr} 3(S, W)$, and Prss19 $(T, X)$ on coronal sections through the hippocampus at low magnification $(A-D, Q-T)$ and high magnification $(E-H, U-X)$. Bisbenzimide staining of the same sections is shown in $I-L$ and $Y-B B$, and gene chip data for each gene are shown in $M-P$ and $C C-F F$. Arrowheads in $E-L$ and $U-B B$ delimit the boundaries of $C A 1$ and also the boundary between $C A 2$ and $C A 3$ in $U-B B$. Scale bars, $1 \mathrm{~mm}$.

Calbindin, a calcium-binding protein well known for its expression in dentate gyrus granule cells (Sequier et al., 1988), is also expressed at moderate levels in CA1 (Fig. $2 X, C C$ ). Interestingly, this pattern of expression is different between mouse and human, where calbindin is expressed in CA2 in addition to CA1 and the dentate gyrus (Maguire-Zeiss et al., 1995). Neuronal guanine nu- 


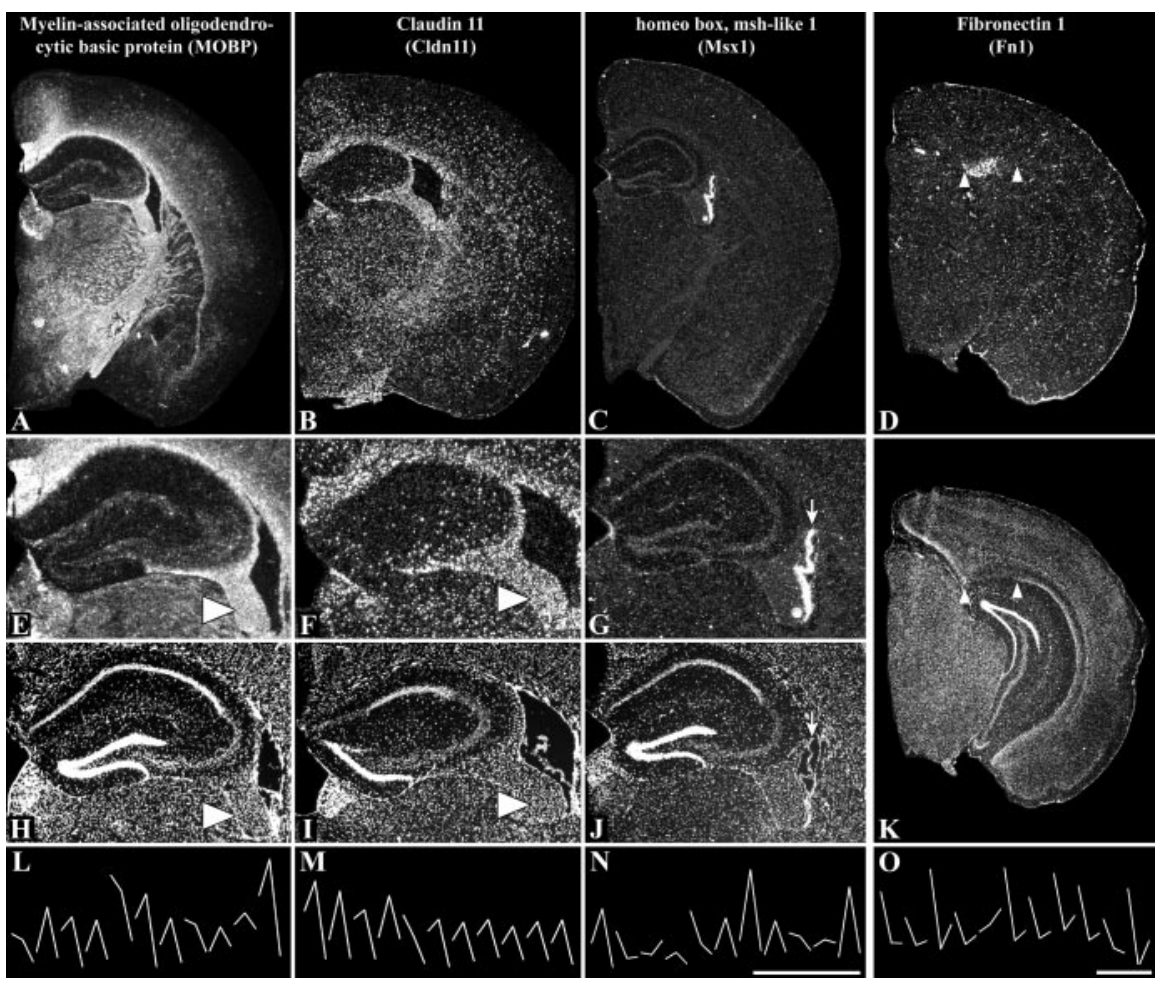

Figure 4. Genes expressed in fimbrial oligodendrocytes, the choroid plexus, and the subiculum. $A-G$, In situ hybridization for $\operatorname{Mobp}(A, E), \operatorname{Cldn11}(B, F), \operatorname{Msx} 1(C, G)$, and $\mathrm{Fn} 1(D)$ on coronal sections through the hippocampus at low magnification $(A-D)$ and high magnification $(E-G)$. Bisbenzimide staining of the same sections is shown in $H-K$, and gene chip data for each gene are shown in $L-0$. Large arrowheads mark the fimbria in $E, F, H$, and $I$, arrows in $G$ and $J$ mark the choroid plexus, and arrowheads delimit the subiculum in $D$ and $K$. Scale bars, $1 \mathrm{~mm}$.

cleotide exchange factor (Fig. 2Y,DD) is also nearly restricted to the dentate gyrus and CA1. Interestingly, no genes were found that were lowest in CA3 alone with high levels in all other hippocampal regions.

\section{Genes enriched in CA1 or lowest in CA1}

A relatively small set of genes could be classified as restricted to or enriched in the pyramidal layer of CA1 (Fig. 3) (supplemental Fig. 6; available at www.jneurosci.org). As described above, expression of Nov is completely restricted to CA1 relative to other hippocampal regions (Fig. 3A,E) (Zhao et al., 2001), although it is also expressed in the neocortex and amygdala and at high levels in the subiculum (data not shown). Several other genes, including the transcription factor Etv1 (Fig. $3 B, F$ ), are enriched in CA1 with relatively uniform expression in the dentate gyrus, CA3, and CA2.

Another series of genes displays patterns best described as lowest in CA1 (or highest in the dentate gyrus plus CA2 plus CA3) (Fig. 3) (supplementary Fig. 7; available at www.jneurosci.org). These include transforming growth factor $\beta 2$ (Fig. $3 C, G$ ) and frizzled-related protein (Fig. $3 D, H$ ). Most genes with this pattern were identified as being enriched in CA3 (Fig. 3O,P).

\section{Genes enriched in the dentate gyrus plus CA2 or lowest in the} dentate gyrus plus CA2

A number of genes was enriched in the dentate gyrus and CA2 (Fig. 3) (supplemental Fig. 8; available at www.jneurosci.org). As described above, Purkinje cell protein 4 (Fig. 3Q,U) is restricted to dentate gyrus granule cells and CA2 pyramidal cells, serving as an excellent marker for the boundaries of CA2 (Zhao et al., 2001).
Tiam1 (Fig. $3 R, V$ ) is also highly enriched in the dentate gyrus with much lower but notable expression in CA2.

The converse pattern was also observed in several genes, with expression in CA1 and CA3 and no expression in the dentate gyrus and CA2 (Fig. 3) (supplementary Fig. 9; available at www.jneurosci.org). Tyro3 (Fig. $3 S, W$ ) is heavily enriched in CA1 but also is expressed in CA3. Expression of the serine protease Prss19 (neuropsin) (Fig. 3T,X) has a similar pattern, although expression levels in CA1 and CA3 are approximately equal.

\section{Genes predicted to be enriched in} hippocampal subregions but actually expressed in neighboring structures Unexpectedly, a fairly large proportion of genes that were predicted to be enriched in CA3 was not expressed at significant levels in primary hippocampal cells at all (Fig. 4) (supplemental Fig. 10; available at www. jneurosci.org). However, these genes were consistently expressed at high levels in two structures adjacent to CA3 throughout the entire septotemporal axis of the hippocampus. Myelin-associated oligodendrocyte basic protein (Fig. $4 A, E$ ) and claudin 11 (Fig. $4 B, F$ ) are expressed at very high levels in oligodendrocytes in the hippocampal fimbria. In contrast, msxl appeared to be expressed solely in the choroid plexus throughout the entire brain (Fig. 4C,G). Expression in these structures was only seen in genes that were predicted to be highest in CA3, indicating that tissue from these structures was included in the CA3 dissection.

Similar to the nonhippocampal expression observed within the CA3-enriched data set, many genes predicted to be highest in CA1 were not expressed in primary hippocampal neurons at significant levels but were instead enriched in the neighboring subiculum (Fig. 4) (supplemental Fig. 11; available at www.jneurosci.org). Fibronectin is the extreme example of this expression pattern, being expressed almost exclusively in the dorsal subiculum and, to a lesser extent, in pial cells and blood vessels (Fig. 4D). A number of the strongest hits for CA1 reflected either expression within both CA1 and the subiculum or just within the subiculum, perhaps indicating that subicular neurons are molecularly more distinct from hippocampal neurons than different types of hippocampal neurons are from one another.

Of the 104 genes tested by in situ hybridization, four genes demonstrated patterns that were at odds with the microarray data. Two of these were predicted to be highest in the dentate gyrus but were not expressed in the hippocampus at all. Tyrosinase-related protein (GenBank accession number X03687) is expressed only in the medial habenula, whereas osteotesticular protein tyrosine phosphatase (GenBank accession number U36488) is expressed only in the striatum and a midline thalamic nucleus (data not shown). Because the habenula in particular is immediately adjacent to the dentate gyrus, it is possible that there was contaminating habenular tissue in the dentate gyrus dissection. Two other genes, the serine protease Prss25 (GenBank accession number AA239856) and an EST (GenBank accession number 


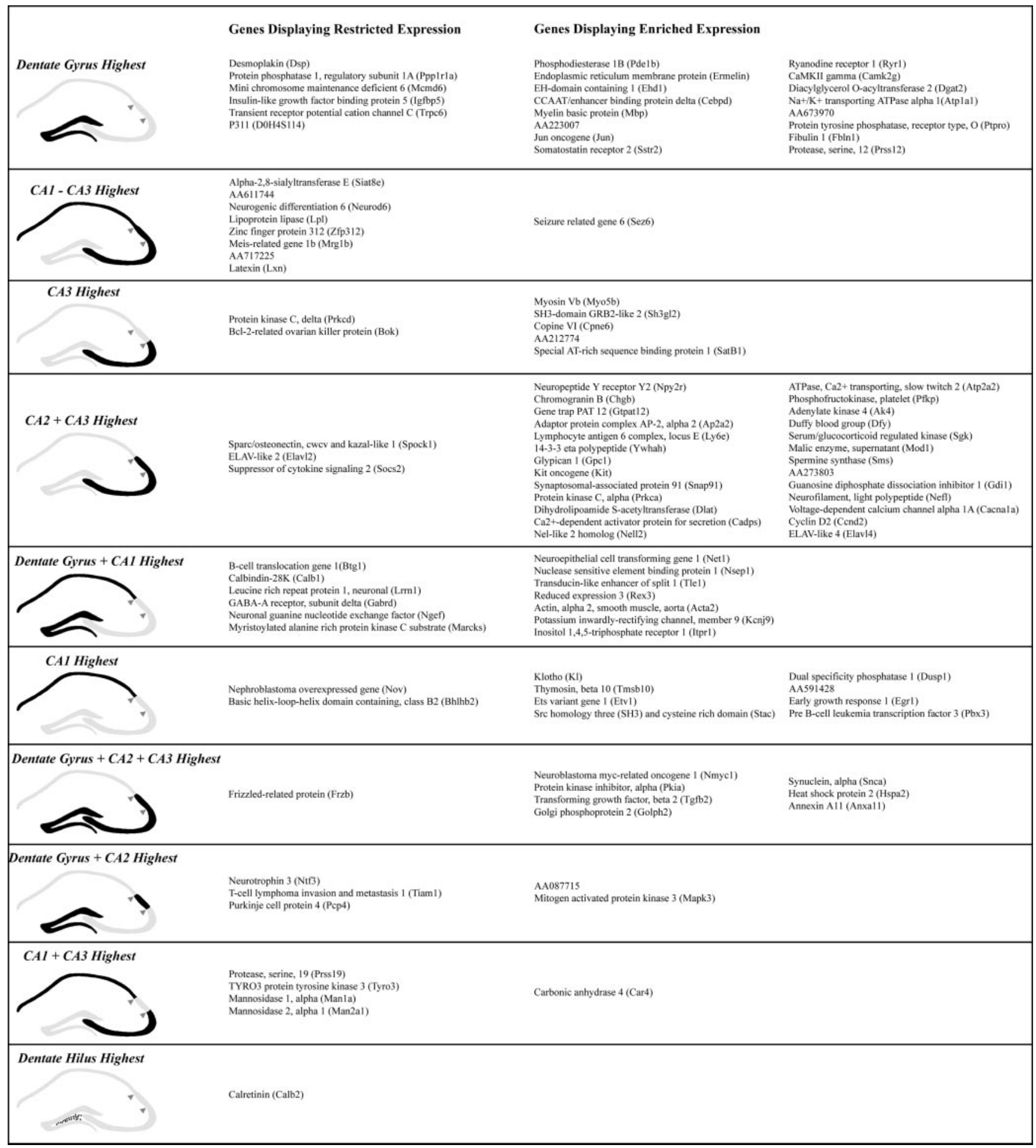

Figure 5. Summary of expression patterns for genes enriched in hippocampal subregions. Genes are clustered into categories on the basis of their expression in primary excitatory neurons in the dentate gyrus, CA1, CA2, CA3, and, in one case, the dentate hilus. A distinction is made between genes with restricted patterns of expression versus those with enriched patterns. Arrowheads in all schematics denote area $\mathrm{CA} 2$.

Z31278), had uniform expression throughout the hippocampus. Although it is possible that the Affymetrix probe sets and our probes correspond to alternatively spliced transcripts, it seems likely that these are true false positives.

To assess the types of genes displaying enriched expression in one or more hippocampal subregions, each gene was assigned a functional classification on the basis of GO classifications (Table
1). The most heavily represented functional categories involved signal transduction and transcriptional regulation. Other heavily represented categories included ion channels, structural molecules, and genes involved in energy metabolism. Among genes with restricted patterns of expression, a significant proportion included growth factors, calcium-ion-binding proteins, and enzymes involved in modification of sugar residues on proteoglycans. 
Table 1. Functional clustering of genes restricted to or enriched in specific hippocampal subregions

\begin{tabular}{llll}
\hline Functional category & Restricted & Enriched & Total \\
\hline Signal transduction & 5 & 13 & 18 \\
Regulation of transcription & 4 & 9 & 13 \\
Ion channels/transporters & 2 & 7 & 9 \\
Cytoskeletal/structural molecules & 3 & 5 & 8 \\
Energy metabolism & 0 & 7 & 7 \\
Growth factors or growth factor-binding proteins & 3 & 2 & 5 \\
Calcium ion-binding proteins & 3 & 1 & 4 \\
Carbohydrate metabolism & 3 & 1 & 4 \\
Extracellular matrix proteoglycans & 2 & 2 & 4 \\
Proteases/protease inhibitors & 2 & 1 & 3 \\
Receptor tyrosine kinases/phosphatases & 1 & 2 & 3 \\
G-protein-coupled receptors & 0 & 3 & 3 \\
RNA-binding proteins & 1 & 1 & 2 \\
Vesicle-mediated transport & 0 & 2 & 2 \\
Secretory granule proteins & 0 & 2 & 2 \\
Endocytosis/endosomal proteins & 0 & 2 & 2 \\
Other or unknown & 8 & 15 & 23 \\
\hline
\end{tabular}

The entire data set is presented in supplemental Table 1 (available at www.jneurosci.org), including average difference values and median FCs from the microarray data as well as a qualitative ranking of in situ hybridization expression within the different hippocampal subregions and surrounding structures. This information allows a very direct comparison of microarray data to actual expression data. Also included for each gene is a synopsis of the reason that the gene matched the predicted pattern and a final classification of the hippocampal expression of that gene (e.g., dentate gyrus enriched, CA1 enriched). Genes falling into each of these categories are summarized in Figure 5.

\section{Discussion}

In the present study, we combined microarray analysis of dissected hippocampal subregions with high-throughput in situ hybridization to verify expression patterns of 104 genes predicted to have enriched (or decreased) levels within hippocampal regions CA1, CA3, or the dentate gyrus. One hundred of these genes demonstrated patterns of gene expression consistent with the initial microarray data, albeit not necessarily in the predicted cell populations. By far, the majority of unpredicted results were attributable to dissection artifacts. A number of genes predicted to be expressed in one region was found to be expressed in neighboring structures, namely the subiculum for genes associated with CA1 and the choroid plexus and fimbria for genes predicted to be expressed in CA3. Furthermore, in a number of cases, gene expression in nonprimary cell populations generated a hybridization signal that was indistinguishable from expression in the primary cell population. For example, calretinin, which was predicted to be expressed in the dentate gyrus, was expressed only in hilar interneurons and not in the more numerous granule cell population. These results demonstrate that microarrays can be used to assess differences in gene expression between different brain regions with tremendous fidelity (at least when large numbers of replicates are used) but that verification of candidate genes to obtain cellular resolution gene expression profiles is essential.

\section{Gene expression and hippocampal cytoarchitecture}

The overwhelming feature that emerged from examination of such a large number of gene expression patterns is the degree to which cytoarchitectural boundaries within the hippocampus are respected. A small set of genes displayed tremendously delimited patterns of expression that could be called restricted to the dentate gyrus, CA3, or CA1, providing unique identifiers for these regions (Zhao et al. 2001). In addition to genes with expression that is restricted to one region, virtually every combination of regions was also observed. From a numerical point of view, the largest sets of genes displaying restricted patterns of expression were in either the dentate gyrus granule cells alone or in all pyramidal cells to the exclusion of the dentate gyrus. This finding might have been expected, given the obvious phenotypic differences between these cell types (Ramon y Cajal, 1911; Lorente de Nó, 1934). However, other patterns observed do not follow such obvious relationships and demonstrate a complex mosaic of gene expression that gives each cell type its unique phenotype in a combinatorial manner.

It is a reasonable assumption that brain regions demonstrating consistently distinct gene expression are functionally distinct as well. It was somewhat surprising that the CA2 subregion, which was not isolated for independent analysis, emerged as a very distinct molecular entity. CA2 forms a narrow band of large pyramidal cells between CA1 and CA3 that can be differentiated from neighboring regions on the basis of synapse morphology (Lorente de Nó, 1934; Claiborne et al., 1986), susceptibility to damage caused by insults and disease (Babb et al., 1984; Akai and Yanagihara, 1993; Maguire-Zeiss et al., 1995), and the expression of several proteins (Williams et al., 1996; Vigers et al., 2000). Nevertheless, the distinctiveness of CA2 has often been discounted; it is sometimes described as an intermingling of cells from CA1 and CA3 (Tole et al., 1997), and in many respects CA2 neurons are indistinguishable from those of CA3. At the level of gene expression, CA2 and CA3 are most often coupled as well. However, in many cases, gene expression in CA2 is distinct from that in CA3 (and CA3 from CA2), indicating that CA2 is a functionally distinct hippocampal subregion [see Woodhams et al. (1993) for a review of this controversy].

\section{Functional correlates of hippocampal subregion-specific genes}

A major aim of genome-wide screens of gene expression in discrete neuroanatomical regions is to search for candidate molecules to account for phenotypic characteristics of particular cell types. Because the candidates in the current screen were chosen solely on the basis of tissue specificity, these results allow an unbiased examination of the functional categories of genes displaying subregion-specific patterns of expression. The largest functional categories for both heavily and moderately enriched genes include molecules involved in signal transduction and transcriptional regulation. Other well represented categories include ion channels and transporters, cytoskeletal or structural proteins, and growth factors (or growth factor-interacting proteins). In general, there was little difference in the functional categories between genes restricted to and enriched in particular areas, with a few notable exceptions. Six of seven genes involved in energy metabolism were ubiquitously expressed but relatively enriched in areas CA2 and CA3. Pyramidal neurons in CA2 and CA3 are the largest neurons in the hippocampus, so this pattern may be a reflection of the greater metabolic needs of larger cells. In contrast, a greater proportion of calcium ion-binding proteins and enzymes involved in modification of carbohydrate moieties on proteoglycans displays highly restricted patterns of expression. Together, however, these data suggest that the distinction made here between highly restricted and enriched gene expression does 
not have a functional correlate and, by extrapolation, that moderate differences in gene expression may be functionally relevant.

The hippocampus is best known for its role in learning and memory and is the model system of choice for studying cellular mechanisms of synaptic plasticity. A number of genes described here play roles in various forms of synaptic plasticity, and the cellular localization of gene transcripts is consistent with the particular synapses involved. For example, mice deficient for calbindin, a calcium-buffering protein expressed in the dentate gyrus and CA1, display impaired spatial learning and maintenance of long-term potentiation (LTP) at Schaffer collateral synapses onto CA1 neurons (Molinari et al., 1996). Neurotrophin 3, restricted to the dentate gyrus and CA2, is specifically involved in perforant path plasticity and not Schaffer collateral plasticity (Kokaia et al., 1998; Ma et al., 1999). Similarly, there is a specific deficit in perforant path LTP in mice lacking protein phosphatase inhibitor-1, which is normally expressed only in the dentate gyrus (Allen et al., 2000). Therefore, the precise cellular localization of genes involved in synaptic plasticity has great predictive power regarding the cellular site of action of that gene.

Hippocampal neurons can also be distinguished from one another on the basis of morphological and physiological criteria. These characteristics are presumably attributable to expression of cytoskeletal proteins and ion channels, a number of which display striking region-specific expression. For example, desmoplakin, known as a component of desmosomal plaques (Kowalczyk et al., 1996), is expressed solely in the dentate gyrus. Granule cell axons, the so-called "mossy fibers," have a very distinctive morphology with extremely large terminal endings characterized by rows of puncta adherens (Claiborne et al., 1986; Acsady et al., 1998). It is tempting to speculate that desmoplakin may function similarly in granule cells, linking intermediate filaments to membrane proteins at mossy fiber synapses. Similarly, a number of ion channels shows differential expression in the hippocampus. For example, the $\mathrm{GABA}_{\mathrm{A}}$ receptor $\delta$ subunit is heavily enriched in the dentate gyrus, and mice lacking this subunit have faster IPSP decay constants in granule cells (Spigelman et al., 2003).

The dentate gyrus has also been studied extensively as a site of ongoing adult neurogenesis (Altman and Das, 1965; Kempermann et al., 1997). Cell division in the SGZ gives rise to new granule neurons throughout adulthood. A number of molecules corresponding to this phenomenon are enriched in the SGZ, including markers for dividing cells (Ki-67) (Kee et al., 2002) and newborn neurons (Doublecortin) (Kempermann et al., 2003). However, none of these SGZ-enriched genes were detectable in the dentate gyrus by microarray, and few of the genes identified as enriched in granule cells are of obvious relevance for cell division or neurogenesis. Several genes that we examined were enriched in the SGZ (see seizure-related gene 6 in supplemental Fig. 3; available at www.jneurosci.org), although they had been selected as genes enriched in other hippocampal subregions. It is likely that genes directly involved in adult neurogenesis are expressed in very restricted populations of cells specifically in the SGZ, and the microarray sensitivity was not sufficient to detect gene expression in such small populations of cells. A more fruitful approach to finding these genes would be to use laser capture to isolate cells in the SGZ (Luo et al., 1999; Kamme et al., 2003) and characterize them independently of the overlying mature granule neurons.

In summary, the use of microarrays to genetically profile different cell types in the hippocampus has been an extremely successful approach to finding differences in gene expression defining these cell types. Large scale in situ hybridization is a feasible method to examine genes on a scale to match microarray data and reveals a tremendously complex mosaic of gene expression defining different hippocampal cell types. The genes identified provide extraordinary markers for these cell types as well as candidate molecules to account for functional differences between these cells. Furthermore, the specificity of these patterns of gene expression lends itself to genetic manipulation of specific cell types using the promoters of these genes. A high-resolution molecular atlas of the hippocampus thus lays the foundation for a wide variety of future studies.

\section{References}

Acsady L, Kamondi A, Sik A, Freund T, Buzsaki G (1998) GABAergic cells are the major postsynaptic targets of mossy fibers in the rat hippocampus. J Neurosci 18:3386-3403.

Akai F, Yanagihara T (1993) Identity of the dorsal hippocampal region most vulnerable to cerebral ischemia. Brain Res 603:87-95.

Allen PB, Hvalby O, Jensen V, Errington ML, Ramsay M, Chaudhry FA, Bliss TV, Storm-Mathisen J, Morris RG, Andersen P, Greengard P (2000) Protein phosphatase-1 regulation in the induction of long-term potentiation: heterogeneous molecular mechanisms. J Neurosci 20:3537-3543.

Altman J, Das GD (1965) Autoradiographic and histological evidence of postnatal hippocampal neurogenesis in rats. J Comp Neurol 124:319-335.

Amaral DG, Insausti R (1990) Hippocampal formation. In: The human nervous system (Paxinos G, ed), pp 711-755. New York: Academic.

Appel RD, Bairoch A, Hochstrasser DF (1994) A new generation of information retrieval tools for biologists: the example of the ExPASy WWW server. Trends Biochem Sci 19:258-260.

Ashburner M, Ball CA, Blake JA, Botstein D, Butler H, Cherry JM, Davis AP, Dolinski K, Dwight SS, Eppig JT, Harris MA, Hill DP, Issel-Tarver L, Kasarskis A, Lewis S, Matese JC, Richardson JE, Ringwald M, Rubin GM, Sherlock G (2000) Gene ontology: tool for the unification of biology. The gene ontology consortium. Nat Genet 25:25-29.

Babb TL, Brown WJ, Pretorius J, Davenport C, Lieb JP, Crandall PH (1984) Temporal lobe volumetric cell densities in temporal lobe epilepsy. Epilepsia 25:729-740.

Blake JA, Richardson JE, Bult CJ, Kadin JA, Eppig JT (2003) MGD: the mouse genome database. Nucleic Acids Res 31:193-195

Claiborne BJ, Amaral DG, Cowan WM (1986) A light and electron microscopic analysis of the mossy fibers of the rat dentate gyrus. J Comp Neurol 246:435-458.

Corsellis JA, Bruton CJ (1983) Neuropathology of status epilepticus in humans. Adv Neurol 34:129-139.

Datson NA, van der Perk J, de Kloet ER, Vreugdenhil E (2001) Expression profile of 30,000 genes in rat hippocampus using SAGE. Hippocampus 11:430-444.

Gonchar Y, Burkhalter A (1997) Three distinct families of GABAergic neurons in rat visual cortex. Cereb Cortex 7:347-358.

Kamme F, Salunga R, Yu J, Tran DT, Zhu J, Luo L, Bittner A, Guo HQ, Miller N, Wan J, Erlander M (2003) Single-cell microarray analysis in hippocampus CA1: demonstration and validation of cellular heterogeneity. J Neurosci 23:3607-3615.

Kee N, Sivalingam S, Boonstra R, Wojtowicz JM (2002) The utility of Ki-67 and BrdU as proliferative markers of adult neurogenesis. J Neurosci Methods 115:97-105

Kempermann G, Kuhn HG, Gage FH (1997) More hippocampal neurons in adult mice living in an enriched environment. Nature 386:493-495.

Kempermann G, Gast D, Kronenberg G, Yamaguchi M, Gage FH (2003) Early determination and long-term persistence of adult-generated new neurons in the hippocampus of mice. Development 130:391-399.

Kokaia M, Asztely F, Olofsdotter K, Sindreu CB, Kullmann DM, Lindvall O (1998) Endogenous neurotrophin-3 regulates short-term plasticity at lateral perforant path-granule cell synapses. J Neurosci 18:8730-8739.

Kowalczyk AP, Borgwardt JE, Green KJ (1996) Analysis of desmosomal cadherin-adhesive function and stoichiometry of desmosomal cadherinplakoglobin complexes. J Invest Dermatol 107:293-300.

Lein ES, Hohn A, Shatz CJ (2000) Dynamic regulation of BDNF and NT-3 expression during visual system development. J Comp Neurol 420:1-18.

Liu G, Loraine AE, Shigeta R, Cline M, Cheng J, Valmeekam V, Sun S, Kulp D, Siani-Rose MA (2003) NetAffx: affymetrix probesets and annotations. Nucleic Acids Res 31:82-86. 
Lockhart DJ, Winzeler EA (2000) Genomics, gene expression and DNA arrays. Nature 405:827-836.

Lorente de Nó R (1934) Studies on the structure of the cerebral cortex. II. Continuation of the study of the ammonic system. J Psychol Neurol (Lpz) 46:113-177.

Luo L, Salunga RC, Guo H, Bittner A, Joy KC, Galindo JE, Xiao H, Rogers KE, Wan JS, Jackson MR, Erlander MG (1999) Gene expression profiles of laser-captured adjacent neuronal subtypes. Nat Med 5:117-122.

Ma L, Reis G, Parada LF, Schuman EM (1999) Neuronal NT-3 is not required for synaptic transmission or long-term potentiation in area CA1 of the adult rat hippocampus. Learn Mem 6:267-275.

Maguire-Zeiss KA, Li ZW, Shimoda LM, Hamill RW (1995) Calbindin D28k mRNA in hippocampus, superior temporal gyrus and cerebellum: comparison between control and Alzheimer disease subjects. Brain Res Mol Brain Res 30:362-366.

Miettinen R, Gulyas AI, Baimbridge KG, Jacobowitz DM, Freund TF (1992) Calretinin is present in non-pyramidal cells of the rat hippocampus-II. Co-existence with other calcium binding proteins and GABA. Neuroscience 48:29-43.

Mody M, Cao Y, Cui Z, Tay KY, Shyong A, Shimizu E, Pham K, Schultz P, Welsh D, Tsien JZ (2001) Genome-wide gene expression profiles of the developing mouse hippocampus. Proc Natl Acad Sci USA 98:8862-8867.

Molinari S, Battini R, Ferrari S, Pozzi L, Killcross AS, Robbins TW, Jouvenceau A, Billard JM, Dutar P, Lamour Y, Baker WA, Cox H, Emson PC (1996) Deficits in memory and hippocampal long-term potentiation in mice with reduced calbindin D28K expression. Proc Natl Acad Sci USA 93:8028-8033.

Ramon y Cajal S (1911) Histologie du Système Nerveux de L'Homme et des Vertébrés, vol II. Paris: Maloine.

Scoville WB, Milner B (2000) Loss of recent memory after bilateral hippocampal lesions. J Neuropsychiatry Clin Neurosci 12:103-113.
Sequier JM, Hunziker W, Richards G (1988) Localization of calbindin D28 mRNA in rat tissues by in situ hybridization. Neurosci Lett 86:155-160.

Spigelman I, Li Z, Liang J, Cagetti E, Samzadeh S, Mihalek RM, Homanics GE, Olsen RW (2003) Reduced inhibition and sensitivity to neurosteroids in hippocampus of mice lacking the GABA(A) receptor delta subunit. J Neurophysiol 90:903-910.

Tole S, Christian C, Grove EA (1997) Early specification and autonomous development of cortical fields in the mouse hippocampus. Development 124:4959-4970.

Vigers AJ, Baquet ZC, Jones KR (2000) Expression of neurotrophin-3 in the mouse forebrain: insights from a targeted LacZ reporter. J Comp Neurol 416:398-415.

Williams TE, Meshul CK, Cherry NJ, Tiffany NM, Eckenstein FP, Woodward WR (1996) Characterization and distribution of basic fibroblast growth factor-containing cells in the rat hippocampus. J Comp Neurol 370:147-158.

Wodicka L, Dong H, Mittmann M, Ho MH, Lockhart DJ (1997) Genomewide expression monitoring in Saccharomyces cerevisiae. Nat Biotechnol 15:1359-1367.

Woodhams PL, Celio MR, Ulfig N, Witter MP (1993) Morphological and functional correlates of borders in the entorhinal cortex and hippocampus. Hippocampus 3:303-311.

Zhao X, Lein ES, He A, Smith SC, Aston C, Gage FH (2001) Transcriptional profiling reveals strict boundaries between hippocampal subregions. J Comp Neurol 441:187-196.

Zirlinger M, Kreiman G, Anderson DJ (2001) Amygdala-enriched genes identified by microarray technology are restricted to specific amygdaloid subnuclei. Proc Natl Acad Sci USA 98:5270-5275.

Zola-Morgan S, Squire LR, Amaral DG (1986) Human amnesia and the medial temporal region: enduring memory impairment following a bilateral lesion limited to field CA1 of the hippocampus. J Neurosci 6:29502967. 